\title{
Color Image Segmentation using CIELab Color Space using Ant Colony Optimization
}

\author{
Seema Bansal \\ SUSWEC, Tangori, \\ Punjab, India
}

\author{
Deepak Aggarwal \\ Asst.Professor \\ BBSBEC, Fatehgarh Sahib \\ Punjab, India
}

\begin{abstract}
Image segmentation plays vital role to understand an image. Only proper understanding of an image tells that what it represents and the various objects present in the image. In this paper we have proposed a new approach by using CIELab color space and Ant based clustering for the segmentation of color images. Image segmentation process divides an image into distinct regions with property that each region is characterized by unique feature such as intensity, color etc. This paper elaborates the ant based clustering for image segmentation. $\mathrm{CMC}$ distance is used to calculate the distance between pixels as this color metric gives good results with CIELab color space. Results shows the segmentation performed using ant based clustering and also shows that number of clusters for the image with particular $\mathrm{CMC}$ distance also varies. In order to evaluate the performance of proposed technique, MSE (Mean Square Error) is used. MSE is the global quality measure based on pixel difference. To verify our work, we have compared the results with results of color image quantization using LAB color model based on Bacteria Foraging Optimization [13].
\end{abstract}

Keywords: Ant Clust, CMC distance, CIELab color space, segmentation.

\section{INTRODUCTION}

Image segmentation process divides an image into distinct regions with property that each region is characterized by unique feature such as intensity, color etc. The objective of segmentation [9] is to simplify and/or change the representation of an image into something that is more meaningful and easier to analyze. Image segmentation is used to visualize objects and boundaries present in an image. Image segmentation is a technique which uniquely identifies pixels which share certain visual characteristics. All the segments generated by the image segmentation process collectively give original image.

Color image segmentation algorithms are based on one of the two basic properties [14]: discontinuity and similarity. In the first case, segmentation is performed on the basis of sharp changes of intensity such as edge where as in the second case we divide an image into regions which are similar with respect to a specific feature. Clustering based image segmentation can be supervised which requires human participation to decide the clustering phenomena and the unsupervised clustering where the clustering phenomenon is decided by itself [16].

\subsection{Lab color Model}

Color is a powerful descriptor in image segmentation that simplifies object identification and extraction from a scene. Color models facilitate the specification of a color in a standard way. A subspace with in a color model gives a single point to represents the color. CIELab color model is perceptual uniform color model where L component of color model represents the human perception of lightness and a,b components represents an amount of a color present. CMC distance measure gives better results with Lab color model [13]. A significant difference between two points in a Lab model using CMC distance metric is represented closely by Euclidean distance measure.

\subsection{Ant Based Clustering}

Image segmentation based on ant clustering was introduced by Deneubourg et al.[10]. ACO is a Meta-heuristic that can be used to refine methods applicable to a wide set of problems with few modifications. The Ant-based clustering algorithms are based upon the brood sorting behavior of ants [12]. In basic model, pixels of the image or data items to be clustered are placed on two dimensional grid. Ants introduced by model, move randomly on the grid for the purpose of picking and dropping data items. The probability of picking and dropping is random and is affected by data items present in the neigbourhood. The drop up probability of an item increases when it is surrounded by high number of similar data items. The pick-up probability increases when the ant carrying data item is surrounded by different data items or when no data is present all around.

The probability of picking and dropping are given by:

Picking up probability:

$$
P_{p}=\left(\frac{k_{1}}{k_{1}+f}\right)^{2}
$$

Dropping Probability

$$
P_{d}=\left(\frac{f}{k_{2}+f}\right)^{2}
$$

Where

F represents a similarity measure in the neighborhood $\mathrm{k}_{1}$ represents picking-up threshold $\mathrm{k}_{2}$ represents dropping threshold 
Short-term memory notion is introduced with each agent by Lumer and Faieta[10]. Small numbers of locations are remembered by an ant where an ant has dropped an item in the previous iterations. When an ant is picking a new item, then ant consults the memory to decide the direction to which the ant will move. Ant's tendency is to move always in the direction where it has most recently dropped a similar kind of data item.

\section{Some of the distinctive features of the Ant based clustering are [15]:}

Solutions of the ant based algorithms are constructed by adding solution components to partial solutions. The main idea behind ant based clustering is that ants communicate indirectly. Ant based algorithms can adopt continuously even if the graph dynamically changes. ACO also clearly differs from BBO, because ACO generates a new set of solutions with each iteration and on the other side, BBO maintains its set of solutions from one iteration to the next, relying on migration to probabilistically adapt those solutions.

\section{LITERATURE SURVEY}

Various techniques available in literature for image segmentation[9] are: gray level thresholding, MRF based approaches, Neural network based approaches, surface based segmentation, Segmentation of color images, segmentation based on edge detection, Methods based on fuzzy set theory. Image pre-processing using image mask is proposed that shortened processing time more than three times [11]. Contrast information [6] of a color image is used to detect edges instead of commonly used derivative information and this new algorithm gives reasonable and reliable results for color image segmentation. Space contraction transformations are introduces into standard Ant Colony System algorithm [7] to increase the speed and to improve the search ability of algorithm. Performance of techniques [4]: Taylor expansion, Iterative procedures and look up table are investigated in terms of speed and accuracy for approximating the nonlinear function in transformation from RGB to CIELab color space. Paper concludes that for real time inspection of color, look up table approach is best. Image segmentation is performed on the basis of color features [1] with K-means clustering unsupervised algorithm. No training data is used. The results shows that proposed scheme reduces the computational cost and gives a high discriminative power of regions present in the image. [5] Reviews a segmentation method based on CIELab color space model and also compares various edge detection methods. The results show that algorithm based on CIELab is appropriate for the color images with various types of noises and from various edge detection methods canny method is most powerful. Clustering with swarm-based algorithms has recently been shown to produce good results in a wide variety of real-world applications [10].ACO algorithm for the segmentation of brain MR images can effectively segments the fine details [8]. By taking advantage of the improvements introduced in ant colony system, edge detection techniques on the basis of ACO was able to successfully extract edges from a digital image[2]. Standard ant based clustering technique is modified in [12]. The algorithm does not require any knowledge of the number of clusters and initial partition during clustering. Results show that the algorithm was able to extract the number of clusters with good quality.
From the literature survey, we concluded to work on ant clustering technique using CIELab color space as CIELab color space closely matches with the human perception and gives best results and no paper has been found with work using similar technique.

\section{PROPOSED ALGORITHM}

ACO is a meta-heuristic where primary goal of the ants is the survival of whole colony. In antclust algorithms, ants move on the $2 \mathrm{D}$ board. In our work, we are replacing the rectangular grid by an array of $\mathrm{N}$ cells where $\mathrm{N}$ is the number of pixels in the image to be clustered. All cells of the array are connected to each other to let the ants travel. During the algorithm, clusters of pixels are created. A cluster is a group of 2 or more pixels with the similar characteristics.

Initially, pixels to be clustered are placed on the array such that each array cell can only be occupied by one pixel. This domain is considered as the cluster space for ant based clustering. With this cluster space, a single agent is placed on a random data item. Then it searches for the neighbor which is uncovered. After finding the uncovered data item, algorithm checks for the similarity. If data item is found with the similar characteristics, then algorithm marks that data item as covered .Once a run is over for an agent, the cluster space is checked for uncovered data items. If any uncovered data item is found then the next ant is introduced and ant finds its cluster as similar procedure. The entire procedure is repeated till there is no uncovered data item. Similarity between the pixels is determined using CMC distance.

For two colors of respective CIELab components (L1, a1, b1) and (L2, a2, b2), CMC metrics define three components for the distance measure as follows:

Chroma difference:

$$
\Delta \mathrm{C}=\sqrt{\mathrm{a} 1^{2}+\mathrm{b} 1^{2}}-\sqrt{\mathrm{a} 2^{2}+\mathrm{b} 2^{2}}
$$

Lighting difference: $\Delta \mathrm{L}=\mathrm{L} 1-\mathrm{L} 2$

Hue perceptual difference: $\Delta \mathrm{H}=\sqrt{\Delta \mathrm{a}^{2}+\Delta \mathrm{b}^{2}-\Delta \mathrm{C}^{2}}$

With the global distance given by:

$$
\Delta \mathrm{E}=\sqrt{\left(\frac{\Delta \mathrm{H}}{\mathrm{SH}}\right)^{2}+\left(\frac{\Delta \mathrm{L}}{\mathrm{l} . \mathrm{SL}}\right)^{2}+\left(\frac{\Delta \mathrm{C}}{\mathrm{c} . \mathrm{SC}}\right)^{2}}
$$

1 and $\mathrm{c}$ are application dependent coefficients where 1 parameter for lightness and c for chroma. SH, SL, SC are tolerances for $\Delta \mathrm{H}, \Delta \mathrm{L}$ and $\Delta \mathrm{C}$

The overall procedure of the proposed algorithm can be described as follows:

1. Take an image and convert it to a Lab image.

2. Place all the pixel in a cell of the array

3. Initialize the cluster for the all data items with 0 and their availability with 1 .

4. Initialize the cluster index with 1 . Introduce an ant 
Initialize the ant by choosing a data item randomly and place the ant.

Check for the availability of data item

Assign the current cluster index

for each data item do

If the data item is not covered, calculate similarity measure $\mathrm{S}$ If $\mathrm{S}<\mathrm{T}$

Select threshold measure of similarity $\mathrm{T}$

Add the data item with the current cluster and assign the current cluster index

Move to the next neighbor.

Endif

Endif

End-for

5. If any item in the cluster-space is available Increase the cluster index by 1 Repeat with the next ant

Else break

End if

Repeat: step 4.

\section{EXPERIMENTAL RESULTS}

Experiments are conducted to evaluate the performance of the proposed approach using three test images with different format, Onion, Lena and Lion which are as shown in Figure1.

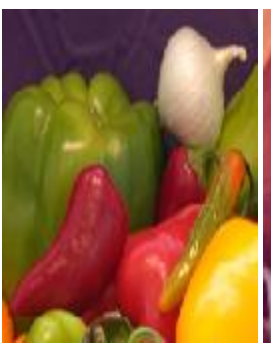

(a)

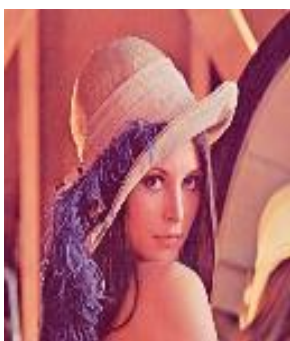

(b)

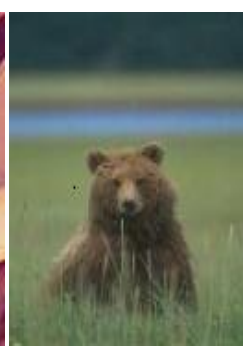

(c)
Fig1. Test images used in this paper (a) Onion.png(128 $\times$ 128); (b) Lena.tiff $(128 \times 128)$; (c) Lion.jpg $(128 \times 128)$

The proposed algorithm automatically calculates the number of clusters on the basis of similarity measure i.e. CMC distance. CMC distance and calculated number of clusters depends on the number of colors present in the image. As the number of colors present in the image increases, CMC distance varies inversely with number of clusters. With the decrease in the CMC distance, number of clusters increases and with the increase in the CMC distance, number of clusters decreases automatically. The proposed algorithm also offers flexibility in calculating the number of clusters with the CMC distance over number of runs because each time it runs, ants are initialized with the different positions, which affect the number of clusters calculation. The proposed algorithm is implemented in Matlab 7.9.0. In order to evaluate the clustering, MSE is taken as a measure and Euclidean distance measure is used to calculate distance between pixels in the cluster.

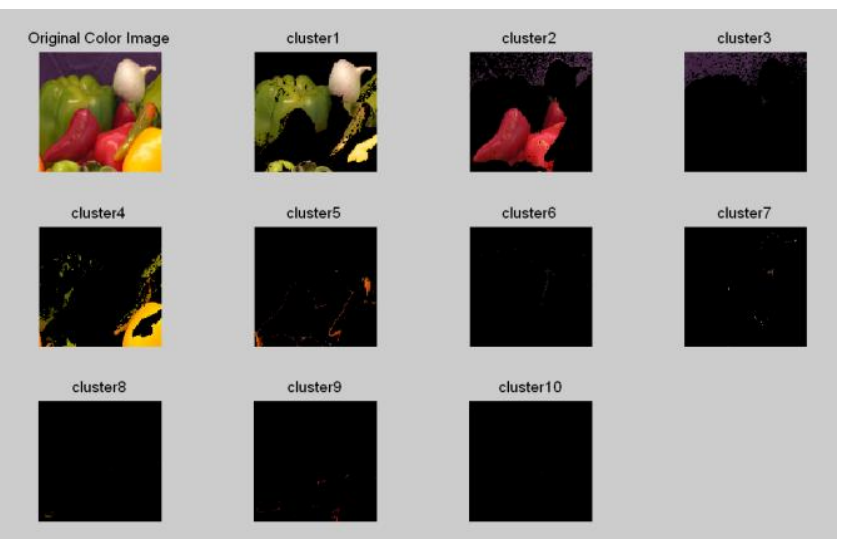

Fig2. Original Onion image with CMC distance 16.7 and 10 no. of clusters

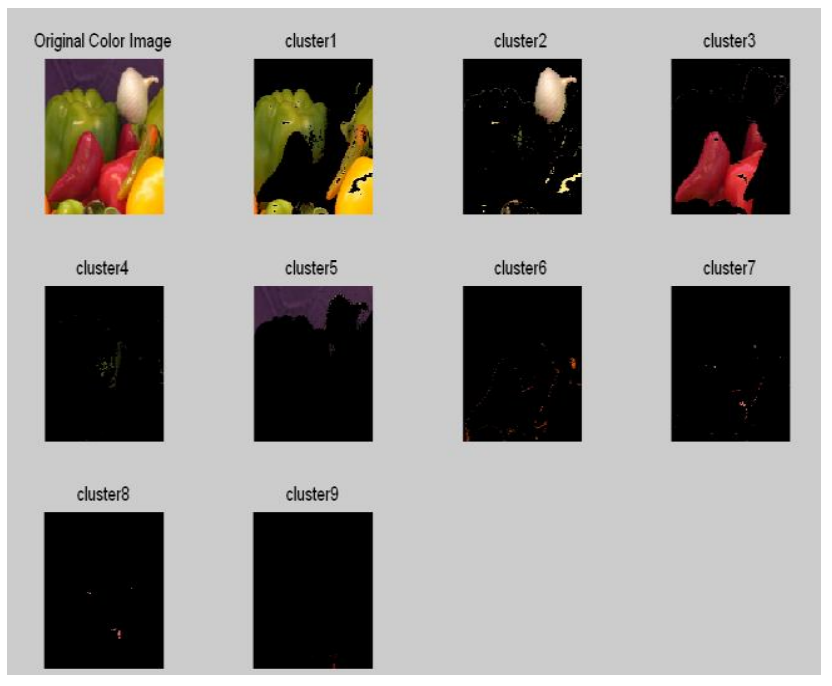

Fig3. Original Onion image with CMC distance 17 and 9 no. of clusters

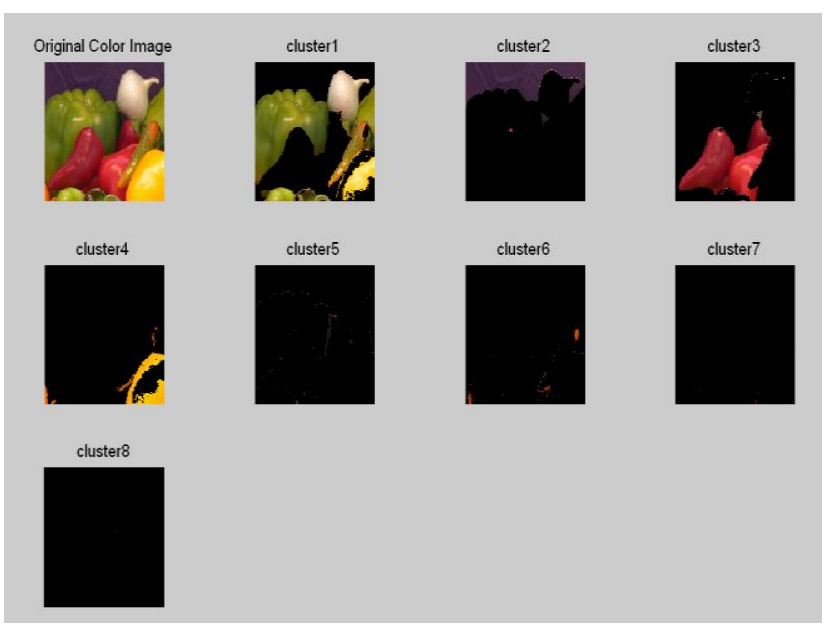

Fig4. Original Onion image with CMC distance 18.5 and 8 no. of clusters 


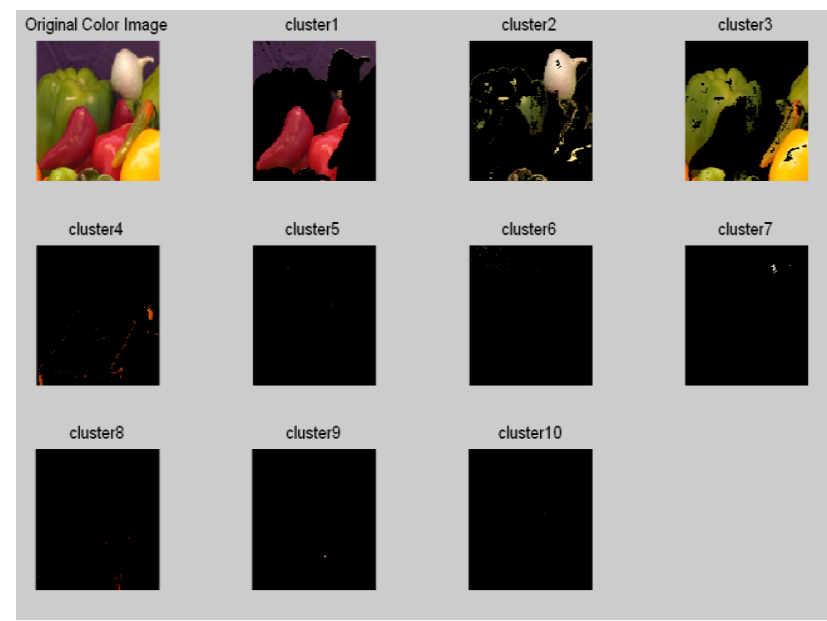

Fig5. Original Onion image with CMC distance 18.5 and 10 no. of clusters

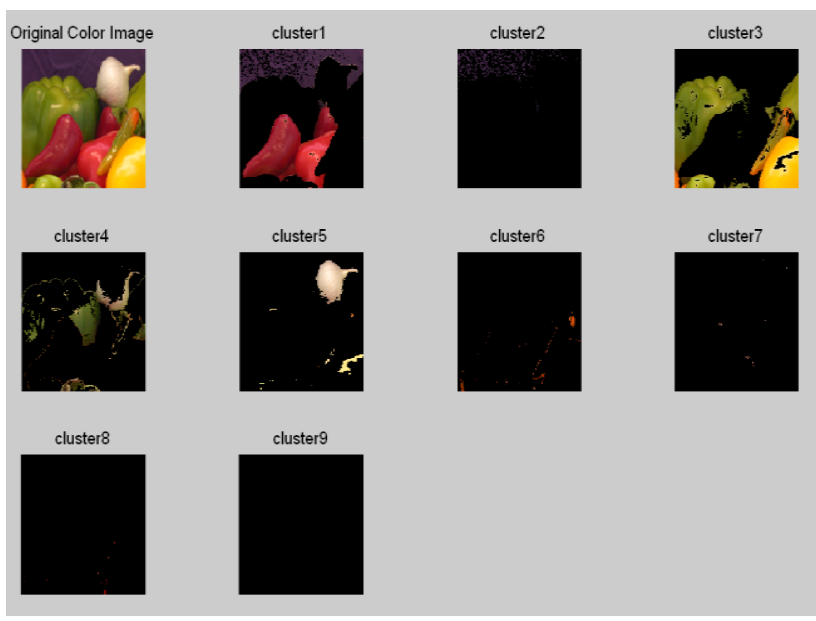

Fig6. Original Onion image with CMC distance 18.5 and 9 no. of clusters

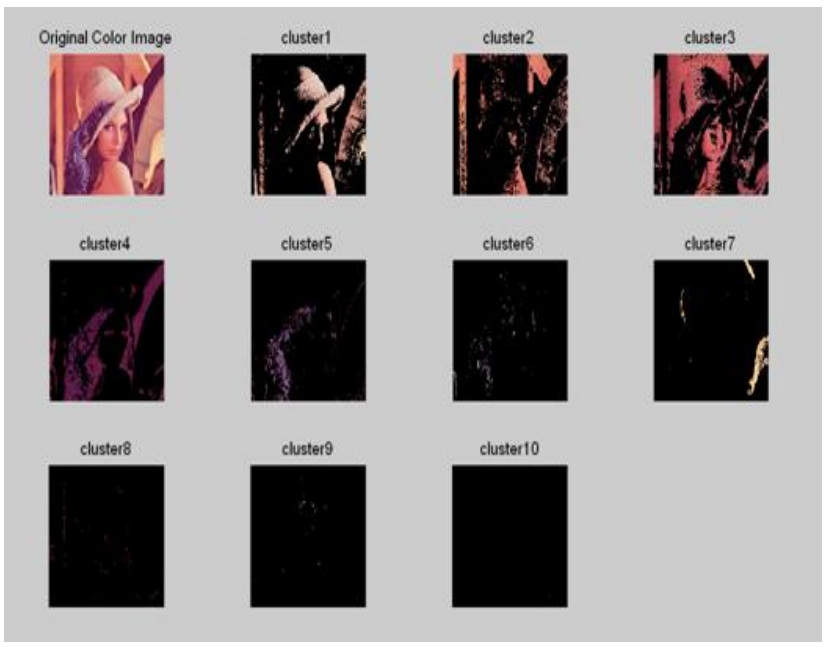

Fig7. Original Lena image with CMC distance 11 and 10 no. of clusters

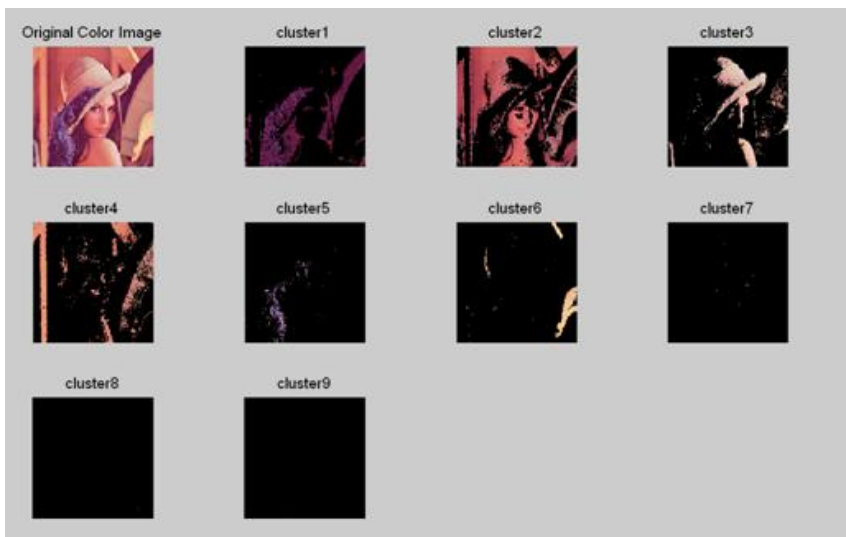

Fig7. Original Lena image with CMC distance 11.5 and 9 no. of clusters

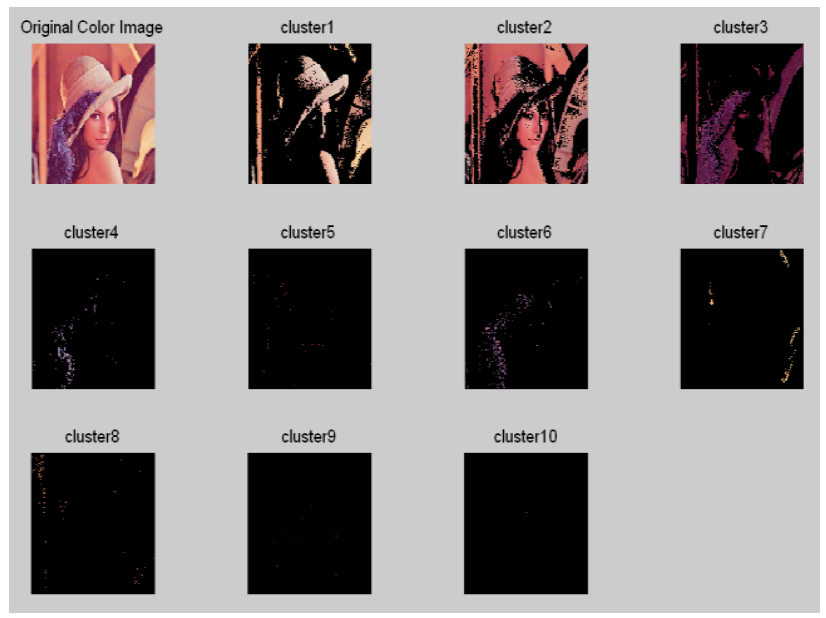

Fig8. Original Lena image with CMC distance 11.5 and 10 no. of clusters

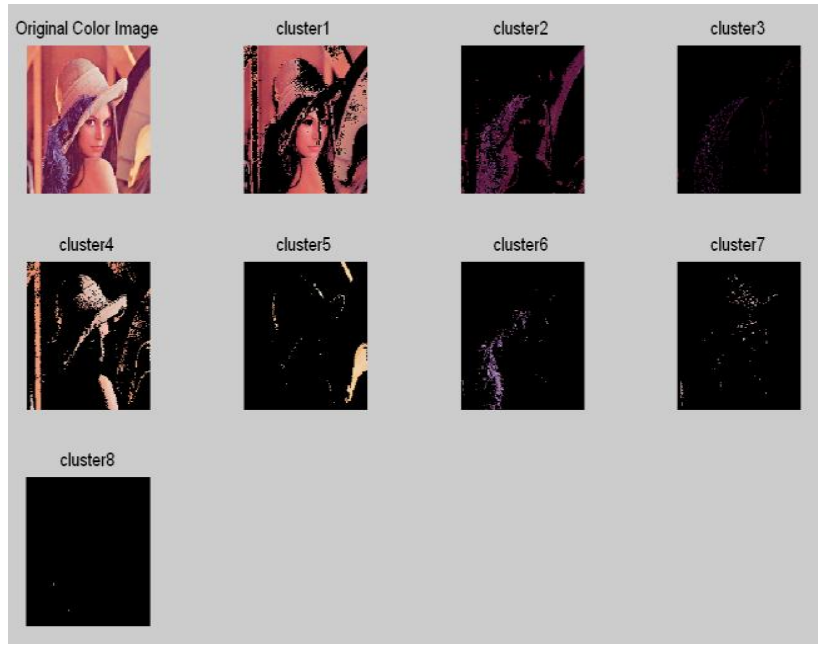

Fig9. Original Lena image with CMC distance 11.5 and 8 no. of clusters 


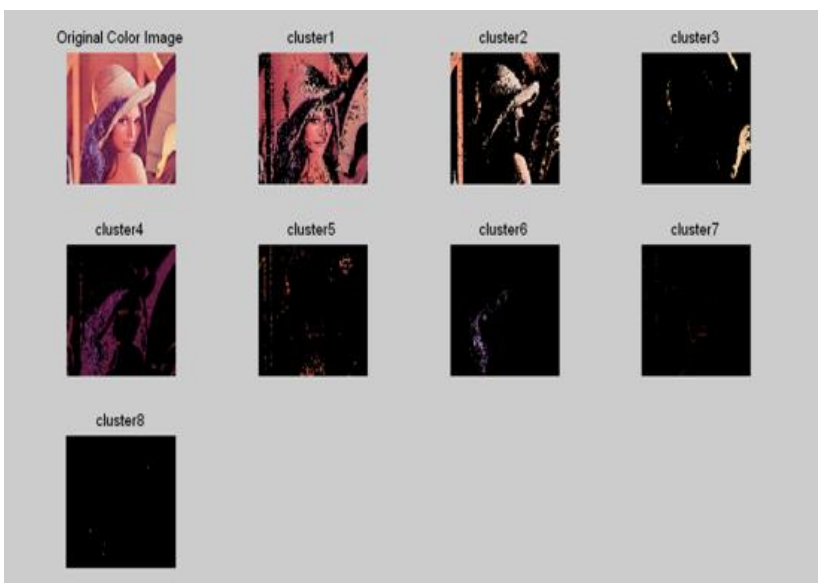

Fig10. Original Lena image with CMC distance 11.7 and 8 no. of clusters

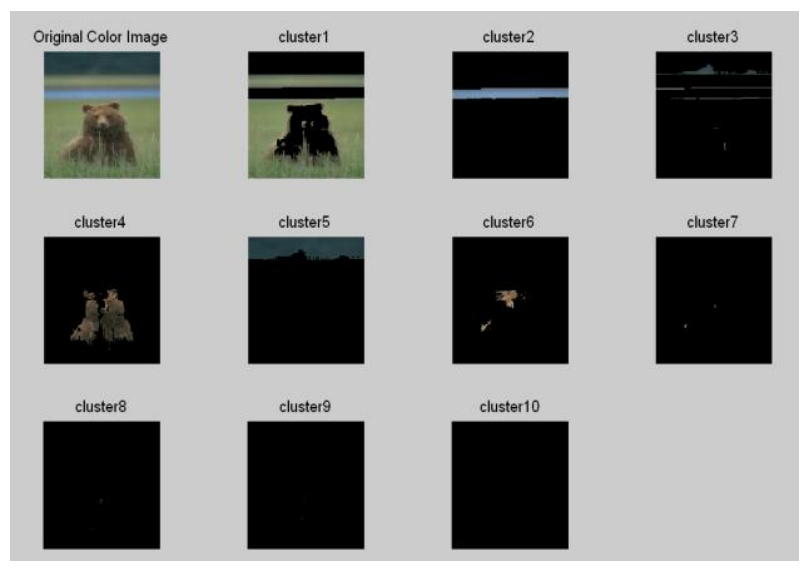

Fig11. Original Lion image with CMC distance 9 and 10 no. of clusters

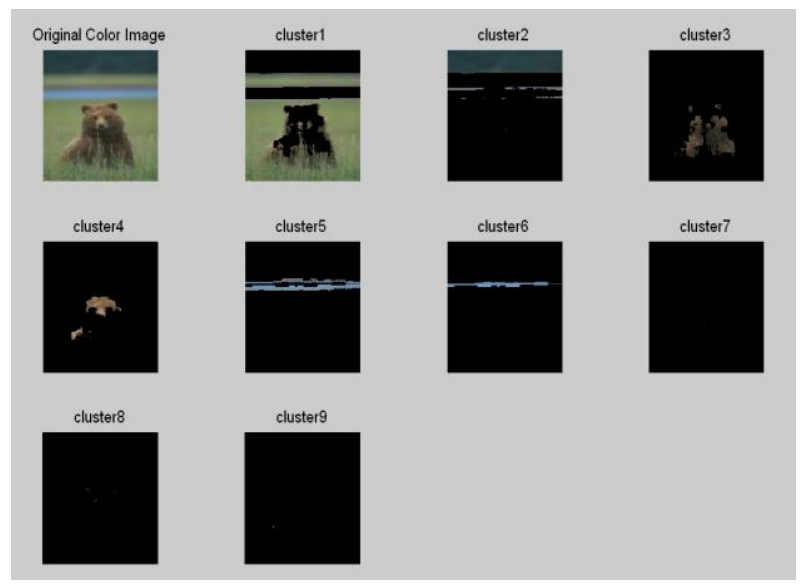

Fig12. Original Lion image with CMC distance 9.3 and 9 no. of clusters

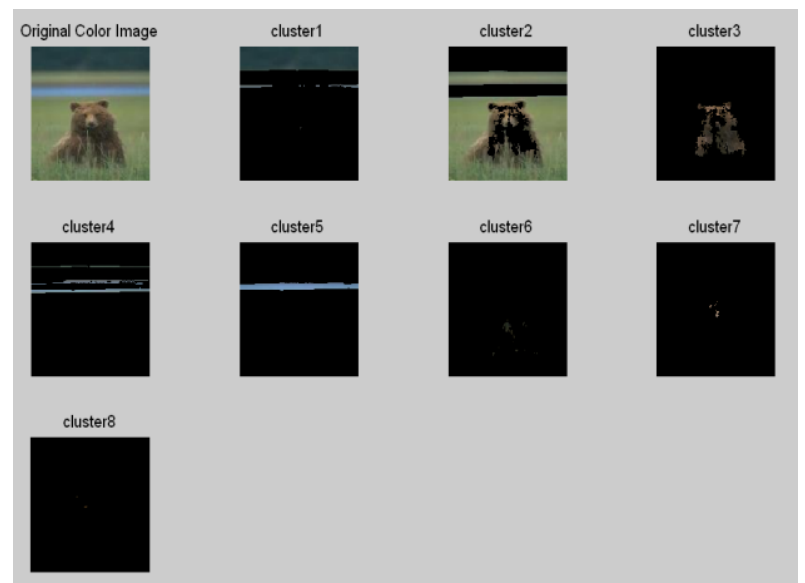

Fig13. Original Lion image with CMC distance 9.4 and 8 no. of clusters

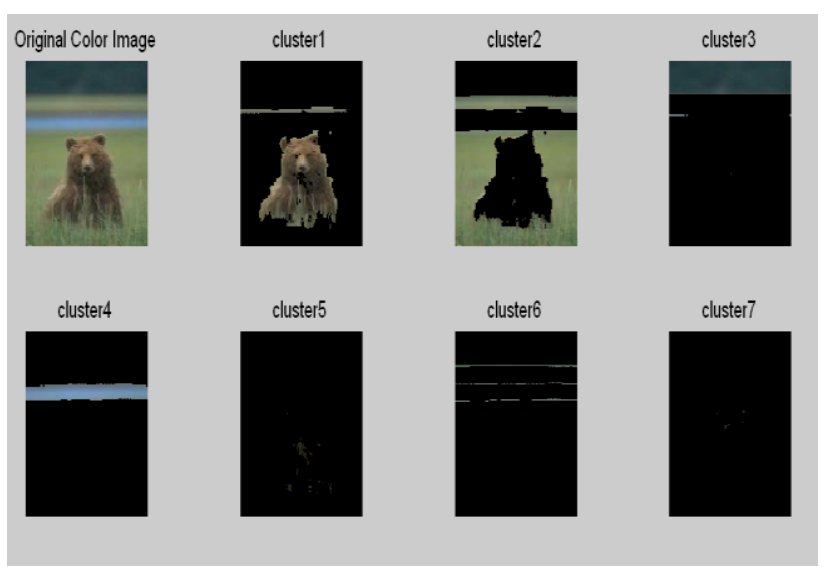

Fig14. Original Lion image with CMC distance 9.4 and 7 no. of clusters

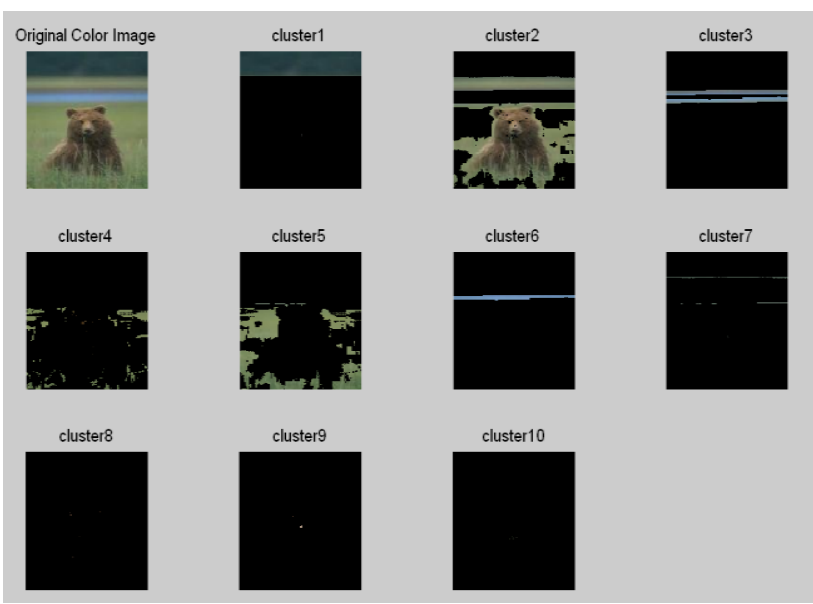

Fig15. Original Lion image with CMC distance 9.4 and 10 no. of clusters 
Table 4. 1. Computational Results

\begin{tabular}{|l|l|l|l|l|}
\hline $\begin{array}{l}\text { Image } \\
\text { Name }\end{array}$ & $\begin{array}{l}\text { No. of } \\
\text { colors }\end{array}$ & $\begin{array}{l}\text { CMC } \\
\text { distance }\end{array}$ & $\begin{array}{l}\text { No. of } \\
\text { clusters }\end{array}$ & MSE \\
\hline Onion.png & 13849 & 16.7 & 10 & 2.5870 \\
\hline Onion.png & 13849 & 17 & 9 & 2.7105 \\
\hline Onion.png & 13849 & 18.5 & 8 & 2.5870 \\
\hline Lena.tiff & 15456 & 11 & 10 & 3.0689 \\
\hline Lena.tiff & 15456 & 11.5 & 9 & 2.4811 \\
\hline Lena.tiff & 15456 & 11.7 & 8 & 2.6710 \\
\hline Lion.jpg & 6359 & 9 & 10 & 2.7176 \\
\hline Lion.jpg & 6359 & 9.3 & 9 & 2.9145 \\
\hline Lion.jpg & 6359 & 9.4 & 8 & 2.9151 \\
\hline
\end{tabular}

Table 4.2. Results shows the variation of number of clusters with particular CMC distance

\begin{tabular}{|l|l|l|l|l|l|}
\hline $\begin{array}{l}\text { Image } \\
\text { Name }\end{array}$ & $\begin{array}{l}\text { No. of } \\
\text { colors }\end{array}$ & $\begin{array}{l}\text { CMC } \\
\text { distance }\end{array}$ & $\begin{array}{l}\text { No. of } \\
\text { clusters } \\
\left(\mathbf{1}^{\text {st }}\right. \\
\text { run) }\end{array}$ & $\begin{array}{l}\text { No. of } \\
\text { clusters } \\
\mathbf{( 2}^{\text {nd }} \\
\text { run) }\end{array}$ & $\begin{array}{l}\text { No. of } \\
\text { clusters } \\
\mathbf{3}^{\text {rd }} \\
\text { run) }\end{array}$ \\
\hline Onion.png & 13849 & 18.5 & 8 & 9 & 10 \\
\hline Lena.tiff & 15456 & 11.5 & 9 & 10 & 8 \\
\hline Lion.jpg & 6359 & $9 . .4$ & 8 & 7 & 10 \\
\hline
\end{tabular}

Table 4.3 Color Image Quantization Results of[13]

\begin{tabular}{|l|l|l|}
\hline $\begin{array}{l}\text { Name of the } \\
\text { Image }\end{array}$ & $\begin{array}{l}\text { Original number of } \\
\text { colors }\end{array}$ & $\begin{array}{l}\text { Colors after } \\
\text { quantization }\end{array}$ \\
\hline Desert.png & 6481 & 4676 \\
\hline Flower1.jpg & 9048 & 5948 \\
\hline Flower2.jpg & 13357 & 8629 \\
\hline Image3.bmp & 15116 & 10489 \\
\hline Lenna.png & 9889 & 5779 \\
\hline
\end{tabular}

From the Table4.1, it can be observed that number of clusters of the image depends on CMC distance selected. The selected $\mathrm{CMC}$ distance in turn depends on the number of colors of the image. Numbers of ants for the purpose are chosen by the algorithm automatically. Number of clusters for the particular $\mathrm{CMC}$ value also varies because the ants are initialized randomly, first time any pixel can be selected which in turn will affect the number of clusters for the image as shown in Table 4.2. From the observation in Table4.1, we come to know that for the onion image where $\mathrm{CMC}$ distance is near 17, Euclidean distance for the pixels in the cluster are near 2.7. It shows how accurate CMC measure is for the Lab color model.

For the verification of work, results of table 4.3 are taken directly from [13]. From the results presented in table4.3, we can easily analyze that the number of colors decreases from 6481 to 4676 with threshold 0.7 for image desert.png. In Our work, threshold value depends on the number of colors. In case the number of colors in image is more, we need to select larger threshold value to get the sufficient number of clusters and in case when number of colors is less, smaller threshold value will give sufficient number of clusters. In our work, we have taken a larger value for CMC to extract the significant number of segments so that we can easily understand the objects present in the image. Above discussion verifies our results. Our algorithm is flexible also as ants are initialized randomly which is the property that is inherited from the general ants behavior.

\section{CONCLUSIONS}

In this paper, an ant based clustering technique using CIELab color space has been successfully developed and tested. Experimental results show the feasibility of the approach in segmentation. With suitable value of $\mathrm{CMC}$, the proposed algorithm was able to successfully segment the test images. It should be noted that the appropriate parameter value depends on the image i.e. number of color in the image. The proposed algorithm also proves the flexibility of the ant clustering approach as the proposed algorithm automatically calculates the number of ants required for the clustering. Number of clusters required to segment the image also varies over number of runs. MSE measure is taken to verify the clustering results.

In the proposed algorithm we are considering each pixel and for large images the proposed algorithm may become slow. So the further research may focus on some modification of the proposed algorithm to enhance the speed. Further research may also focus on developing some new algorithms where present technique is combined with swarm intelligence techniques. Future research may also try to apply the proposed technique to other color spaces.

\section{REFERENCES}

[1] Anil Z Chitade et. al., "COLOUR BASED IMAGE SEGMENTATION USING K-MEANS CLUSTERING”, International Journal of Engineering Science and Technology Vol. 2(10), 2010, 5319-5325.

[2] Anna Veronica Baterina et al., "Image Edge Detection Using Ant Colony Optimization”, INTERNATIONAL JOURNAL OF CIRCUITS, SYSTEMS AND SIGNAL PROCESSING, Issue 2, Volume 4, 2010.

[3] C. Immaculate Mary et al., "A Modified Ant-based Clustering for Medical Data", (IJCSE) International Journal on Computer Science and Engineering Vol. 02, No. 07, 2010, 2253-2257.

[4] Christine Connolly et al., "A Study of Efficiency and Accuracy in the Transformation from RGB to CIELAB Color Space", IEEE TRANSACTIONS ON IMAGE PROCESSING, VOL. 6, NO. 7, JULY 1997.

[5] Ganesan P et al., "Segmentation and Edge Detection of Color Images Using CIELAB Color Space and Edge Detectors", 978-1-4244-9005-9/10/\$26.00 @2010 IEEE.

[6] Hsin-Chia Chen et al., "Contrast-Based Color Image Segmentation", IEEE SIGNAL PROCESSING LETTERS, VOL. 11, NO. 7, JULY 2004. 
[7] Liqiang Liu and HaijiaoRen et al., "Ant Colony Optimization Algorithm Based on Space Contraction", Proceedings of the 2010 IEEE, International Conference on Information and Automation, June 20 - 23, Harbin, China.

[8] Myung-Eun Lee1 et al., "Segmentation of Brain MR Images using an Ant Colony Optimization Algorithm", 978-0-7695-3656-9/09 \$25.00 @ 2009 IEEE.

[9] N.R. Pal et al., "A review on image segmentation techniques”, Pattern Recognition 9(26): 1277-1294, 1993.

[10] O.A. Mohamed Jafar, "Ant-based Clustering Algorithms: A Brief Survey ", International Journal of Computer Theory and Engineering, Vol. 2, No. 5, October, 2010, 1793-8201.

[11] R. Laptik et al., "Application of Ant Colony Optimization for Image Segmentation”, ISSN 1392 - 1215, 2007. No. 8 (80).

[12] Salima Ouadfel et al., "An Efficient Ant Algorithm for Swarm-Based Image Clustering", Journal of Computer
Science 3 (3): 162-167, 2007 ISSN 1549-3636 ㄷ 2007 Science Publications.

[13] Rajinder Kaur et al., "Color Image Quantization based on Bacteria Foraging Optimization", International Journal of Computer Applications (0975 - 8887) Volume 25- No.7, July 2011.

[14] PunamThakare et al., "A Study of Image Segmentation and Edge Detection Techniques", International Journal on Computer Science and Engineering (IJCSE), ISSN : 9753397 Vol. 3 No. 2 Feb 2011.

[15] Surbhi Gupta et al., "Implementing Color Image Segmentation Using Biogeography Based Optimization", 2011 International Conference on Software and Computer Applications IPCSIT vol.9 (2011) @ (2011) IACSIT Press, Singapore.

[16] S. Thilagamani et al., "A Survey on Image Segmentation Through Clustering", International Journal of Research and Reviews in Information Sciences ,Vol. 1, No. 1, March 2011. 